\title{
Lidar systems for precision navigation and safe landing on planetary bodies
}

\author{
Farzin Amzajerdian ${ }^{1}$, Diego Pierrottet ${ }^{2}$, Larry Petway ${ }^{1}$, Glenn Hines ${ }^{1}$, and Vincent Roback ${ }^{1}$ \\ ${ }^{1}$ NASA Langley Research Center, Hampton, Virginia, 23681, United States of America \\ ${ }^{2}$ Coherent Applications, Inc., Hampton, Virginia, 23666, United States of America
}

\begin{abstract}
The ability of lidar technology to provide three-dimensional elevation maps of the terrain, high precision distance to the ground, and approach velocity can enable safe landing of robotic and manned vehicles with a high degree of precision. Currently, NASA is developing novel lidar sensors aimed at needs of future planetary landing missions. These lidar sensors are a 3-Dimensional Imaging Flash Lidar, a Doppler Lidar, and a Laser Altimeter. The Flash Lidar is capable of generating elevation maps of the terrain that indicate hazardous features such as rocks, craters, and steep slopes. The elevation maps collected during the approach phase of a landing vehicle, at about $1 \mathrm{~km}$ above the ground, can be used to determine the most suitable safe landing site. The Doppler Lidar provides highly accurate ground relative velocity and distance data allowing for precision navigation to the landing site. Our Doppler lidar utilizes three laser beams pointed to different directions to measure line of sight velocities and ranges to the ground from altitudes of over $2 \mathrm{~km}$. Throughout the landing trajectory starting at altitudes of about $20 \mathrm{~km}$, the Laser Altimeter can provide very accurate ground relative altitude measurements that are used to improve the vehicle position knowledge obtained from the vehicle navigation system. At altitudes from approximately $15 \mathrm{~km}$ to $10 \mathrm{~km}$, either the Laser Altimeter or the Flash Lidar can be used to generate contour maps of the terrain, identifying known surface features such as craters, to perform Terrain relative Navigation thus further reducing the vehicle's relative position error. This paper describes the operational capabilities of each lidar sensor and provides a status of their development.
\end{abstract}

Keywords: Laser Remote Sensing, Laser Radar, Doppler Lidar, Flash Lidar, 3-D Imaging, Laser Altimeter, Precession Landing, Hazard Detection

\section{INRODUCTION}

Landing mission concepts being developed for exploration of planetary bodies are increasingly ambitious in their implementations and objectives. Most of the missions under consideration by NASA will require precision landing at the pre-designated sites of high scientific values and on-board terrain hazard detection and avoidance capability. Laser remote sensing technologies can play a major rule in enabling these missions. Currently, NASA-LaRC is developing novel lidar landing sensors under the Autonomous Landing and Hazard Avoidance (ALHAT) project ${ }^{1}$. These lidar sensors are 3-Dimensional Imaging Flash Lidar, Doppler Lidar, and Laser Altimeter.

To fulfill the requirements of landing at any pre-designated site under any lighting conditions, ALHAT is pursuing active sensor technology development and maturation to implement five sensor functions: Altimetry, Velocimetry, Terrain Relative Navigation (TRN), Hazard Detection and Avoidance (HDA) and Hazard Relative Navigation (HRN). The three lidar sensor systems noted above can perform these functions with some degree of redundancy. The operation of these advanced lidar sensors may be best described in the context of a lunar landing scenario as shown in Figure 1. As the landing vehicles initiates its powered descent toward the landing site at about $20 \mathrm{~km}$ above the surface, the Laser Altimeter begins its operation providing altitude data with sub-meter precision. This measurement will reduce the vehicle position error significantly since the Inertial Measurement Unit (IMU) suffers from drastic drift over the travel time from the Earth. The IMU drift error can be over $1 \mathrm{~km}$ for a Moon-bound vehicle and over $10 \mathrm{~km}$ for Mars. The accurate altitude data can reduce the position error to a few hundred meters in 
the case of Moon. Shortly after, the Flash Lidar starts its operation by having its laser beam focused to illuminate a subset of its pixels generating a relatively low-resolution elevation data of the terrain below. The reason for reducing the divergence of the lidar transmitter beam to a fraction of its receiver field of view is to increase its operational range to about $15 \mathrm{~km}$ from a nominal $1 \mathrm{~km}$. At this altitude, the Flash Lidar can generate a contour map of the terrain for matching them with on-board maps of known surface features such as craters. This process, referred to as Terrain Relative Navigation, will further reduce the vehicle relative position error from hundreds of meters to tens of meters. When the landing vehicle descends to about $2.5 \mathrm{~km}$, the Doppler lidar initiates its operation by providing ground-relative vector velocity and altitude data with high precision of the order of $1 \mathrm{~cm} / \mathrm{sec}$ and $10 \mathrm{~cm}$, respectively. The Doppler lidar data will allow for precision navigation to the selected landing site to better than a meter precision. From about $1 \mathrm{~km}$ to $0.5 \mathrm{~km}$ from the ground, the Flash Lidar will operate with its full field of view generating a high resolution elevation map of the landing area identifying hazardous features such as rocks, craters, and steep slopes. This elevation map is then processed to determine the most suitable safe landing location (HDA function). Flash lidar continues to generate updated maps of the landing area. The updated maps allows for using the terrain features, such as rocks, as marker for establishing a trajectory toward the selected landing location. This phase of Flash Lidar operation is referred to as Hazard Relative Navigation. The Flash Lidar operation is ceased at about $100 \mathrm{~m}$ above the ground before the vehicle thrusters create a dust plume. The high precision velocity and altitude data provided by the Doppler Lidar allows the Guidance, Navigation, and Control system to direct the vehicle to the identified landing site and ensure a safe and smooth landing.

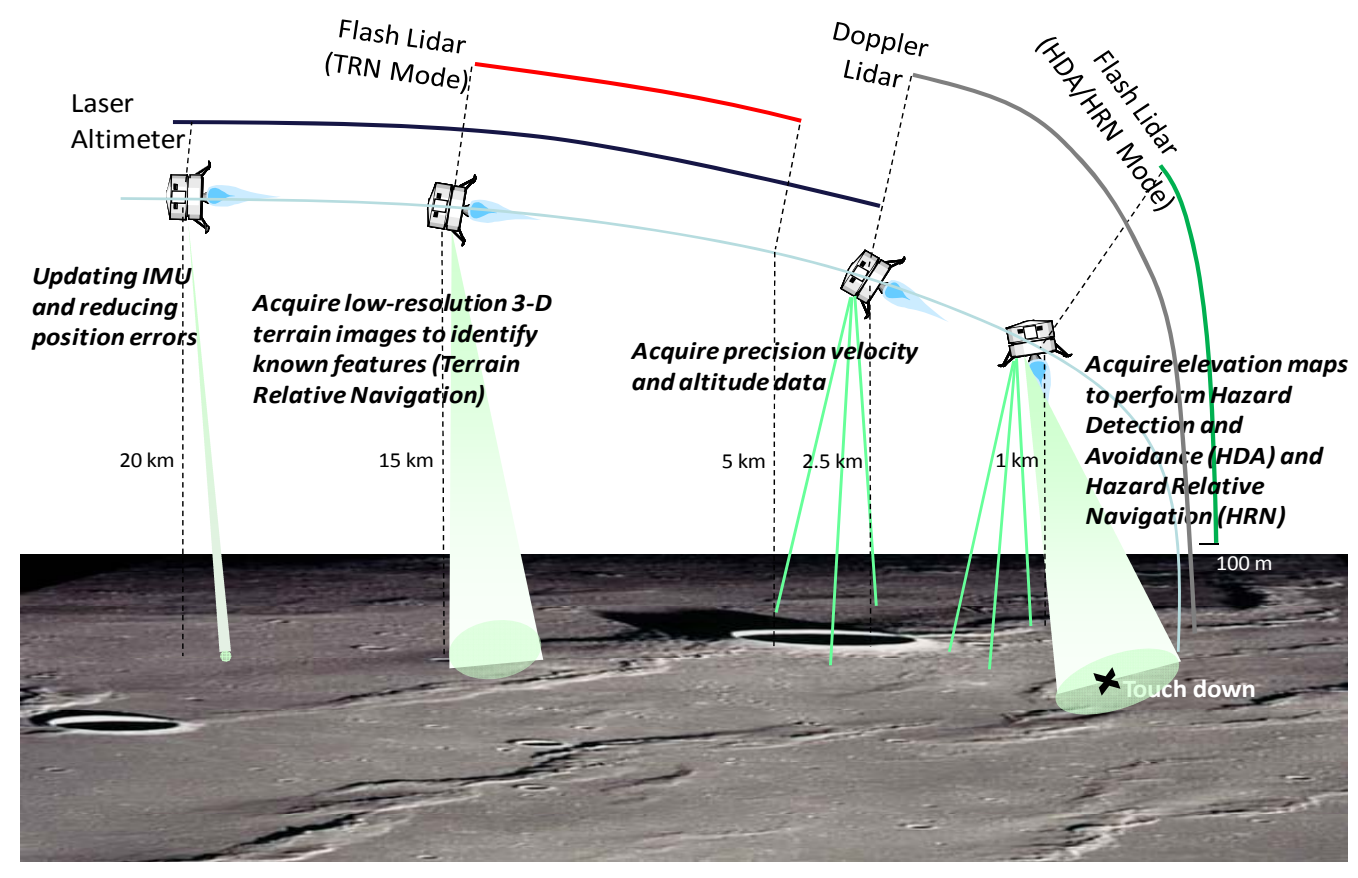

Fig. 1. Operational Scenario of Landing Sensors.

\section{LIDAR SENSORS OPERATIONAL DESCRIPTION}

The performance and operational requirements of the lidar sensors are driven by the ALHAT project goals summarized per following:

- Autonomous operation under any lighting conditions and any landing location

- Global Landing Precision: Landing to within 30 meters of predetermined location in the case of lunar landing

- Hazard Detection: Terrain features greater than $30 \mathrm{~cm}$ in height and slopes greater than 5 degrees over the vehicle footprint $(\sim 10 \mathrm{~m})$ 
- Local Landing Precision: Landing to within $1 \mathrm{~m}$ error at the safe location selected by the onboard Hazard

Detection and Avoidance algorithm

Table 1 below lists the ALHAT sensor suite and their top level performance specifications for performing each of the five required functions with some degree of redundancy. Flash Lidar is capable of performing all the functions with exception of velocimetry for which a Doppler Lidar is being developed. The ability of the Doppler Lidar to provide velocity data with approximately $1 \mathrm{~cm} / \mathrm{sec}$ is highly attractive for precision landing. Additionally, the Doppler Lidar provides high resolution altitude and ground-relative attitude data that may further improve precision navigation to the identified landing site. The Laser Altimeter provides independent altitude data over a large operational altitude range of $20 \mathrm{~km}$ to $100 \mathrm{~m}$. All three laser sensors have a nominal update rate of $30 \mathrm{~Hz}$. Although the Flash lidar is fully capable of providing the necessary altitude data, the Laser Altimeter is used in the operational scenario of Figure 1 in order to lessen spacecraft accommodation issues. Since the orientation of the spacecraft will be different during different phases of descent and landing, it may be difficult to accommodate the Flash Lidar such that it will have a clear view of the ground during the approach phase $(<2 \mathrm{~km}$ altitude), when it needs to perform HAD and HRN functions, as well as powered descent phase $(20 \mathrm{~km}-10 \mathrm{~km}$ altitude), when Altimetry and TRN functions need to be performed. Therefore, the Laser Altimeter can reduce the complexity of spacecraft accommodation design and ease the spacecraft attitude control requirements. Even if the Flash lidar can operate over the entire powered descent phase, the Laser Altimeter may serve as a redundant sensor for either or both Altimetry and TRN functions.

Table 1. ALHAT Sensors Performance Requirements.

\begin{tabular}{|l|l|l|l|}
\hline Sensor & Function & Operational Altitude Range & Precision/Resolution \\
\hline \multirow{3}{*}{ Flash Lidar } & HDA/HRN & $1000 \mathrm{~m}-100 \mathrm{~m}$ & $5 \mathrm{~cm} / 40 \mathrm{~cm}$ \\
\cline { 2 - 4 } & TRN & $15 \mathrm{~km}-5 \mathrm{~km}$ & $20 \mathrm{~cm} / 6 \mathrm{~m}$ \\
\cline { 2 - 4 } & Altimetry & $20 \mathrm{~km}-100 \mathrm{~m}$ & $20 \mathrm{~cm}$ \\
\hline \multirow{2}{*}{ Doppler Lidar } & Velocimetry & $2500 \mathrm{~m}-10 \mathrm{~m}$ & $1 \mathrm{~cm} / \mathrm{sec}$ \\
\cline { 2 - 4 } & Altimetry & $2500 \mathrm{~m}-10 \mathrm{~m}$ & $10 \mathrm{~cm}$ \\
\hline \multirow{2}{*}{ Laser Altimeter } & Altimetry & $20 \mathrm{~km}-100 \mathrm{~m}$ & $20 \mathrm{~cm}$ \\
\cline { 2 - 4 } & TRN $^{1}$ & $15 \mathrm{~km}-5 \mathrm{~km}$ & $20 \mathrm{~cm}$ \\
\hline
\end{tabular}

1 Secondary function, maybe considered as redundancy option.

\section{3-DIMENSIONAL IMAGING FLASH LIDAR}

The capabilities of the Flash Lidar technology for autonomous safe landing application have been investigated through a series of static and dynamic experiments at the NASA-LaRC sensor test range, and from helicopter and fixed-wing aircraft platforms $\mathrm{s}^{2-4}$. All the Flash Lidar experiments to date have been based on the 3-D Imaging camera technology developed by Advanced Scientific Concepts (ASC) ${ }^{5}$. This 3-D Imaging camera has a $128 \times 128$ pixel array capable of generating real-time image frames at up to $30 \mathrm{~Hz}$ rate. In addition to demonstration of Flash Lidar capabilities, these tests served several other important objectives. One of these objectives was to define areas of technology improvement required to meet NASA's autonomous safe and precision landing needs. Another objective was to support the development of algorithms for the HDA, HRN, and TRN functions and analyze their respective performance towards achieving the specified goals. The analyses of data collected from these tests allowed for improving the Flash lidar computer models used in the end-to-end landing system simulations. The tests data also proved invaluable for the development of image reconstruction and enhancement algorithms that can result in generation of elevation maps of the extended landing site from about $1 \mathrm{~km}$ altitude.

Earlier static and airborne tests resulted in initiation of a series of Flash Lidar component technology advancement projects in 2008, conducted in collaboration with industry, aimed at the development of a Flash Lidar landing sensor system that can efficiently perform the ALHAT functions described above. The Flash Lidar component technology projects and their status are summarized in Table 2. These activities include the development of low noise 256x256 pixel Avalanche Photodiode array, high sensitivity 256x256 Readout Integrated Circuit (ROIC), rugged and compact transmitter laser with optimum pulse temporal and spatial profiles, programmable field-of-view receiver optics, and novel signal processing techniques. These technology advancements have resulted in significant increase 
in operational range by more than a factor 3 , higher frame rate, and a much more robust system. Table 3 summarizes the current state of the Flash Lidar technology and the performance goal of ongoing technology advancement activities.

The primary performance requirement of the Flash Lidar is to generate a Digital Elevation Map (DEM) of an area at least $100 \mathrm{~m} \mathrm{X} 100 \mathrm{~m}$ in dimensions from $1000 \mathrm{~m}$ slant range with a Ground Spot Distance (GSD) of $10 \mathrm{~cm}$ and elevation precision of $5 \mathrm{~cm}$. The development of larger format detector and ROIC of 256x256 pixels will help achieving the specified Flash Lidar performance requirements. Current 128X128 pixels camera will require a mosaic of over 100 image frames in order to create the required DEM. With $10 \mathrm{~cm}$ GSD, each frame will cover only $12.8 \mathrm{~m} \mathrm{X} 12.8 \mathrm{~m}$ area and accurate registration of each image frame will require significant overlapping of the image frames. Therefore, the total number of image frames will be larger than $(100 \mathrm{~m} / 12.8 \mathrm{~m})^{2}=61$. The larger format detector and ROIC of 256x256 pixels will reduce the number of frames by a factor 4 thus making the generation of $100 \mathrm{~m}^{2}$ DEM considerably easier and faster. The ongoing development of ROIC will also improve the range measurement precision from current $8 \mathrm{~cm}$ to $5 \mathrm{~cm}$ required for detecting $30 \mathrm{~cm}$ hazards with high reliablity ${ }^{6}$. The 256X256 focal plane array (detector + ROIC) is expected to have higher sensitivity by at least a factor of 4 and half the pitch size compared with current focal plane, thus allowing the use of the same laser and receiver optics.

Table 2. Flash Lidar Component Technologies Advancement.

\begin{tabular}{|l|l|l|l|}
\hline Component Technology & Description & Lead Organization & Status \\
\hline Transmitter laser & $\begin{array}{l}\text { High pulse energy optimized for Flash } \\
\text { Lidar, compact and rugged design suitable } \\
\text { for space }\end{array}$ & Fibertek & Complete \\
\hline Detector Array & $\begin{array}{l}\text { Low noise InGaAs Avalanche Photodiode } \\
\text { Detector array }\end{array}$ & Optogration & Complete \\
\hline $\begin{array}{l}\text { Image reconstruction and } \\
\text { enhancement processor }\end{array}$ & $\begin{array}{l}\text { 3-D Super-resolution algorithm, real-time } \\
\text { implementation in high-speed HDW }\end{array}$ & NASA LaRC & 2012 \\
\hline Fiber optic delivery unit & $\begin{array}{l}\text { High pulse energy fiber cable for } \\
\text { transmitter beam, rugged designed for space }\end{array}$ & NASA GSFC & 2012 \\
\hline 256X256 Sensor Engine & $\begin{array}{l}\text { Low noise HgCdTe APD detector with } \\
\text { advanced ROIC including control/interface } \\
\text { electronics }\end{array}$ & Raytheon & 2012 \\
\hline 256X256 Sensor Engine & $\begin{array}{l}\text { Advanced high sensitivity ROIC hybridized } \\
\text { with Optogration APD array including } \\
\text { control/interface electronics }\end{array}$ & ASC & 2013 \\
\hline Receive/Transmit Optics & Variable Field of View & NASA LaRC & 2013 \\
\hline
\end{tabular}

As an alternative to building a mosaic of large number of image frames for creating a DEM with sufficient dimensions, a novel image resolution enhancement technique is being pursued that can eliminate the need for precision scanning of the lidar and significantly reduce the DEM construction time. The image resolution enhancement can be achieved by means of a super-resolution algorithm in real-time. The super-resolution algorithm is based on the back projection method that has recently been applied to solve the super resolution problem in the 2D environment ${ }^{7}$. Analysis of processed Flash Lidar data indicates that 8 times resolution enhancement is feasible. Study of the processed data also shows reduction in random noise as multiple image frames are blended to create a single high resolution DEM. A real-time super-resolution processor is currently being developed to demonstrate its potentials for landing application.

Table 3. Flash Lidar Current State and Performance Goals.

\begin{tabular}{|l|l|l|}
\hline Parameter & Current & Goal \\
\hline Max operational range & $1400 \mathrm{~m}$ & $1400 \mathrm{~m}$ \\
\hline Number of pixels & $128 X 128$ & $256 X 256$ \\
\hline Field of view & Fixed $1,3,5 \mathrm{deg}$ & Variable $6-24 \mathrm{deg}$ \\
\hline Elevation precision & $8 \mathrm{~cm}$ & $5 \mathrm{~cm}$ \\
\hline Frame update rate & $20 \mathrm{~Hz}$ & $30 \mathrm{~Hz}$ \\
\hline
\end{tabular}




\section{DOPPLER LIDAR}

The Doppler Lidar is a versatile instrument capable of providing precision velocity vectors relative to the sensor reference frame, vehicle platform altitude, and ground relative attitude. With this sensor the landing vehicle can acquire a surface inertial navigation fix during the approach phase, accurate to a few centimeters in position and about one centimeter per second in velocity. This allows the vehicle to accurately navigate from a few kilometers altitude to the previously defined surface location very accurately.

The Doppler Lidar obtains high-resolution range and velocity information from a frequency modulated continuous wave (FMCW) laser waveform whose instantaneous frequency is modulated linearly with time. Figure 2 shows the waveform's frequency content versus time, and the resulting intermediate frequency (IF) that holds the desired range and velocity information. The green triangular waveform represents the frequency content of the transmitted waveform, and the blue trace simulates a received waveform. The horizontal shift to the right of the received waveform is due to the time delay caused by the round trip time of flight of the laser beam to the target. The vertical shift of the received waveform represents the Doppler frequency change that arises from the motion of the vehicle relative to the ground.

The lidar design uses an optical homodyne receiver configuration, in which a portion of the transmitted beam serves as the reference local oscillator (LO) for the optical receiver. The LO optical field mixes with the time delayed received field at the detector yielding a time varying intermediate frequency (IF) as shown by the lower (red) trace in Figure 2. The IF trace shows two distinct frequencies, one caused by the up-ramp, and one caused by the downramp of the waveform. The difference in up-ramp and down-ramp frequency provides the vehicle velocity and their mean value provides the range to the ground. The lidar transmits 3 laser beams separated 45 degrees pointed nadir in order to determine the 3 components of the vehicle velocity, and to accurately measure altitude and attitude relative to the local ground.

A breadboard Doppler Lidar was assembled and tested onboard a helicopter in 2008 to evaluate its capabilities for the landing application. The results of helicopter test showed excellent agreement with the high accuracy GPS derived velocities. The data collected during the flight tests also proved to be very valuable for the development of a compact and efficient system, shown in Figure 3, which was demonstrated in a helicopter flight test campaign in 2010. Examples of this flight test data are provided in Figure 4 where the Doplpler Lidar data is compared with the data from a high grade Inertial Measurement Unit (IMU) and GPS system built by Applanix. The Doppler Lidar data showed excellent

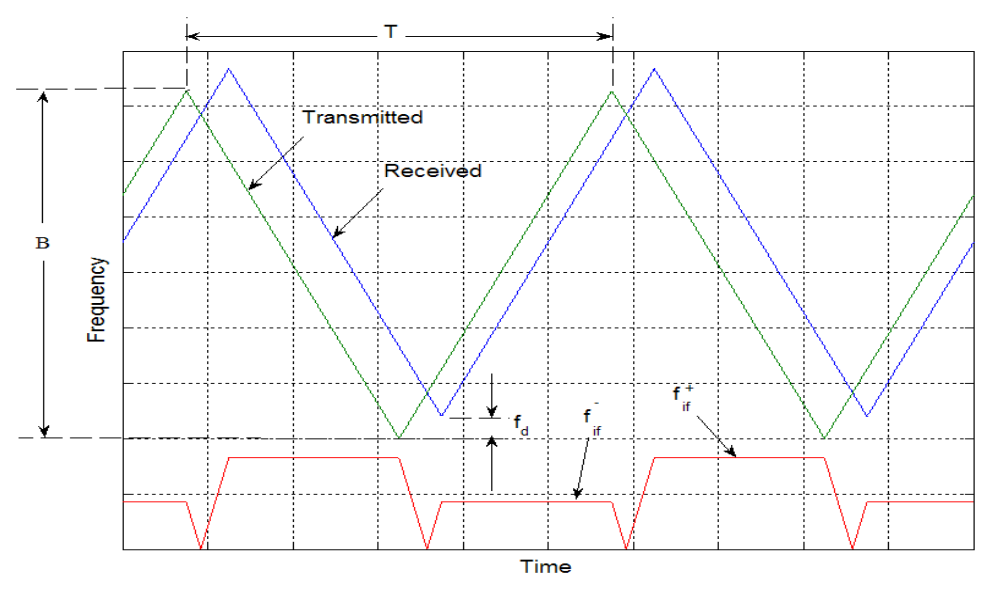

Fig. 2. The laser frequency modulation has a linear chirp waveform. Received waveform is delayed in time. Lower trace is the difference between transmit and receive waveforms. agreement with the IMU/GPS data. With the scale of these plots, the Doppler Lidar and IMU/GPS data essentially overlap and not distinguishable. The mean value of discrepancy in magnitude of the vector velocity measurements was about $3.5 \mathrm{~cm} / \mathrm{sec}$. Quantifying the discrepancies between the Doppler Lidar and IMU/GPS measurements of altitude is not as straight forward since the GPS measures altitude relative to sea level while the Doppler Lidar provides altitude relative to the local ground. For the altitude plot of Figure 4, a fixed bias was subtracted from the GPS data so it can be compared with the above ground level (AGL) altitude measurements of the Lidar. This ground altitude bias was determined from the Lidar data at the beginning of the flight taken over a flat dry lake. The 
discrepancy in altitude measurements is very small and within the scale of the ground surface roughness. A more detailed description of the Doppler Lidar and the results of the latest helicopter test flight are provided in a recent publication $^{8}$.

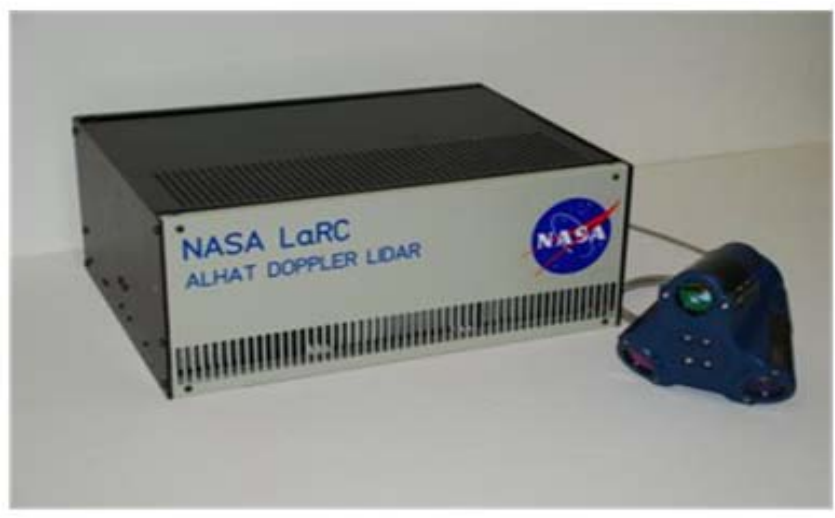

Fig. 3. Doppler Lidar prototype system with a fibercoupled optical head having 3 lenses pointing to different directions. The Doppler Lidar provides vehicle vector velocity and altitude.
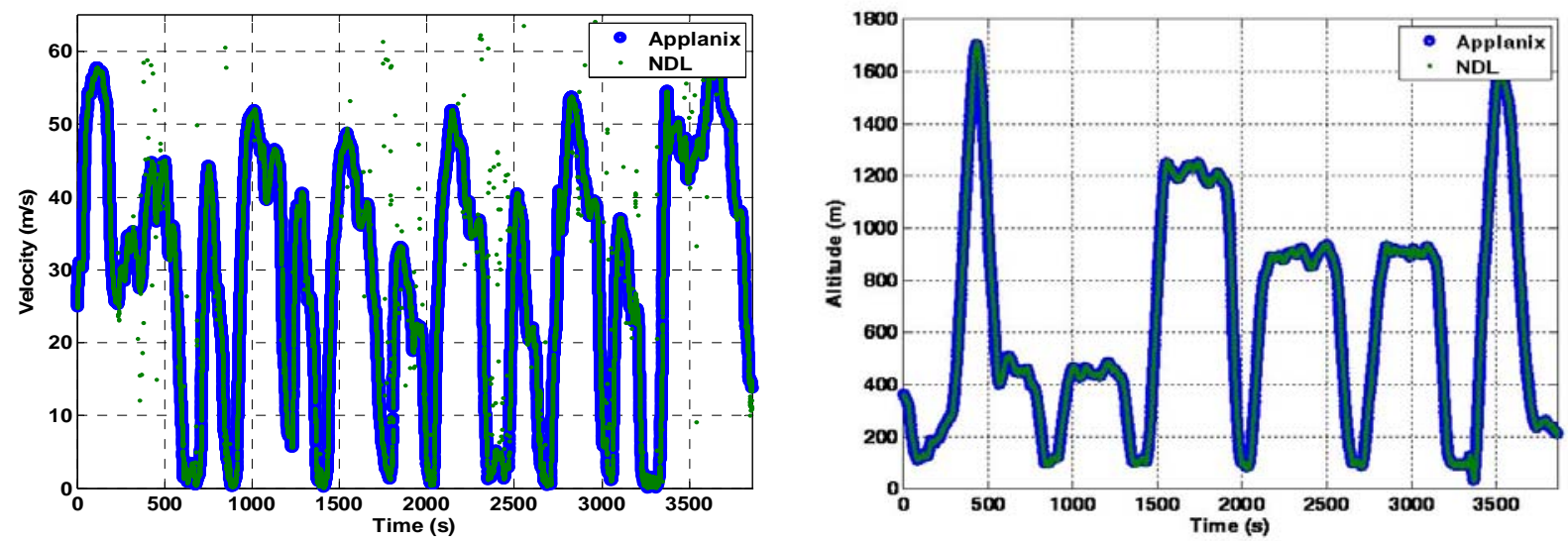

Fig. 4. Example of helicopter flight test data, comparing the magnitude of platform velocity and AGL altitude provided by the Doppler Lidar and a high grade IMU/GPS unit (Applanix).

\section{LASER ALTIMETER}

The vehicle altitude can be measured by the Flash Lidar at high altitudes approaching $20 \mathrm{~km}$ and the Doppler Lidar from altitudes of a few km's above the ground. However, a separate Laser Altimeter sensor can ease the spacecraft accommodation design and provide a redundancy to this critical data. A Laser Altimeter has been designed and built specifically for ALHAT. The breadboard version of this sensor was first tested in the fixed-wing aircraft test from altitudes over $8 \mathrm{~km}$ in 2008. A compact and low-power prototype system, as shown in Figure 5, was recently completed and tested at the NASA-LaRC lidar test range facility. The results of measurements indicated that the Altimeter sensor exceeds the requirements of Table 1 . The measured performance parameters were $30 \mathrm{~km}$ maximum operational range, $8 \mathrm{~cm}$ range precision, and an accuracy of $0.0034 \%$ of range. This system operates at an eye safe energy level at 1.5 micron wavelength for easier terrestrial operation and testing. 


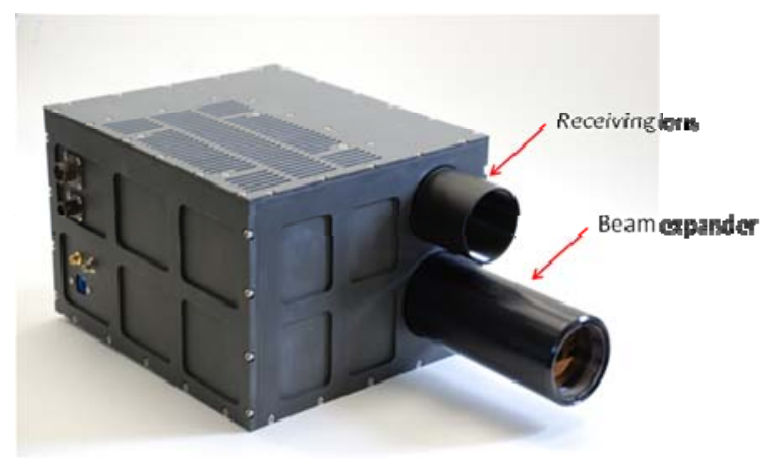

Fig. 5 Laser Altimeter Sensor.

\section{CONCLUSION}

Lidar has been identified by NASA as a key technology for enabling autonomous precision safe landing of future robotic and crewed lunar landing vehicles. NASA-LaRC has been developing three laser/lidar sensor systems under the ALHAT project. The capabilities of these Lidar sensor systems were evaluated through a series of static tests using a calibrated target and through dynamic tests aboard helicopters and a fixed wing aircraft. The airbone tests were perfomed over Moon-like terrain in the California and Nevada deserts. These tests provided the necessary data for the development of signal processing software, and algorithms for hazard detection and navigation. The tests helped identify technology areas needing improvement and guiding ongoing technology advancement activities.

\section{ACKOWLEDGMENTS}

The authors would like to acknowledge the contributions of NASA Jet Propulsion Laboratory ALHAT team for the flight tests support and data processing of the flight trajectories. The authors are also thankful to the ALHAT project manager, Chirold Epp, NASA Johnson Space Center for his support.

\section{REFERENCES}

[1] C. D. Epp, E. A. Robinson, and T. Brady, "Autonomous Landing and Hazard Avoidance Technology (ALHAT)", Proc. of IEEE Aerospace Conference, pp.1-7 (2008).

[2] D. Pierrottet, F. Amzajerdian, B. Meadows, R. Estes, A. Noe, "Characterization of 3-D imaging lidar for hazard avoidance and autonomous landing on the Moon,", Proc. of SPIE Vol. 6550 (2007).

[3] Alexander Bulyshev, Diego Pierrottet, Farzin Amzajerdian, George Busch, Michael Vanek, and Robert Reisse, "Processing of three-dimensional flash lidar terrain images generating from an airborne platform," Proc. SPIE Vol. 7329, (2009).

[4] F. Amzajerdian, M. Vanek, L. Petway, D. Pierrottet, G. Busch, A. Bulyshev, "Utilization of 3-D Imaging Flash Lidar Technology for Autonomous Safe Landing on Planetary Bodies," SPIE Proceeding Vol. 7608, paper no 80 (2010).

[5] R. Stettner, H. Bailey, and S. Silverman, "Three Dimensional Flash Ladar Focal Planes and Time Dependent Imaging," International Symposium on Spectral Sensing Research, Bar Harbor, Maine (2006).

[6] A. Huertas, A. E. Johnson, R. A. Werner, R A. Maddock, "Performance Evaluation of Hazard Detection and Avoidance Algorithms for Safe Lunar Landings," Proc. IEEE Aerospace Conference, PP 1-20 (2010).

[7] Alexander Bulyshev, Michael Vanek, Farzin Amzajerdian, Diego Pierrottet, Glenn Hines, and Robert Reisse, “A super-resolution algorithm for enhancement of FLASH LIDAR data," Proc. SPIE 7873, 78730F (2011).

[8] Diego Pierrottet, Farzin Amzajerdian, Larry Petway, Bruce Barnes, George Lockard, and Glenn Hines, "Navigation Doppler Lidar Sensor for Precision Altitude and Vector Velocity Measurements Flight Test Results." SPIE Defense and Security Symposium, Orlando, FL, (2011). 